\title{
A systematic review of observed climate change adaptation in developed nations
}

\section{A letter}

\author{
James D. Ford · Lea Berrang-Ford • Jaclyn Paterson
}

Received: 16 November 2010 / Accepted: 26 January 2011 / Published online: 5 March 2011

(C) The Author(s) 2011. This article is published with open access at Springerlink.com

\begin{abstract}
We develop and apply a systematic mixed-methods literature review methodology to identify and characterize how climate change adaptation is taking place in developed nations. We find limited evidence of adaptation action. Where interventions are being implemented and reported on, they are typically in sectors that are sensitive to climate impacts, are most common at the municipal level, facilitated by higher-level government interventions, with responses typically institutional in nature. There is negligible description of adaptation taking place with respect to vulnerable groups, with reporting unequal by region and sector. The methodology offers important insights for meta-analyses in climate change scholarship and can be used for monitoring progress in adaptation over time.
\end{abstract}

\section{Introduction}

Adaptation is necessary if we are to manage the risks posed by climate change (New et al. 2011; Smith et al. 2011). What we know about adaptation, however, is limited. While there is an emerging scholarship proposing assessment approaches and adaptation options, few studies have systematically examined actual adaptation actions at a national or regional level. Is adaptation taking place? What types of interventions are being implemented? What factors are motivating adaptation? The assessment reports of the IPCC contain information on these questions, but using the IPCC to examine, compare, and monitor if and how adaptation is taking place presents a number of challenges. While IPCC assessments are rigorous and comprehensive, critiques have highlighted the opacity of selection criteria for literature included

Electronic supplementary material The online version of this article (doi:10.1007/s10584-011-0045-5) contains supplementary material, which is available to authorized users.

J. D. Ford $(\varangle) \cdot$ L. Berrang-Ford · J. Paterson

Department of Geography, McGill University, Montreal, H3A2K6, Canada

e-mail: james.ford@mcgill.ca 
and excluded (Berrang-Ford et al. 2011; Ford and Pearce 2010). Thus chapter 17 of working group II (WGII) profiles examples of adaptation action but it is unclear if these actions represent a comprehensive list of actions being implemented or what criteria was used for selection of examples. The detail at which actions are discussed also varies throughout WGII: in some instances the purpose, timing, scope, function, and form of adaptation actions are specified, for others less information is provided. This reflects the design of the IPCC to provide a broad overview of our knowledge on climate change, not answer specific questions; an objective which has guided climate change meta-analyses more generally (e.g. national assessments, NAPAs). As the climate change field evolves, however, new literature review approaches are needed if we are to identify and characterize what we know, don't know, and need to know (Hulme 2009; Hulme et al. 2010).

In this paper we develop a systematic mixed-methods review methodology to examine if and how adaptation is taking place in developed nations. The methodology advances existing approaches to meta-analysis and allows us to critically examine how adaptation is taking place. We define 'developed nations' as the 41 Parties identified under Annex I to the UNFCCC (see Supplementary Data S1).

\section{Methodology}

\subsection{Systematic reviews}

A systematic literature review is a summary and assessment of the state of knowledge on a given topic or research question, structured to rigorously summarize existing understanding. Neglected in climate change research but long established in the health sciences, systematic reviews differ from traditional literature reviews in three main ways (Ford and Pearce 2010). Firstly, they involve reviewing documents according to clearly formulated questions (e.g. are we adapting?) and using systematic and explicit methods and criteria to select relevant research. Secondly, they involve full reporting of search terms and criteria for inclusion and exclusion of articles, documenting publications excluded in the analysis along with those included. This contrasts to literature reviews common in climate change research which typically do not provide detail on the review procedures employed (e.g. databases searched, articles excluded, search terms used); in absence of such detail, it is difficult to replicate the study to validate interpretation and examine comprehensiveness. Finally, systematic selection of articles permits the use of quantitative and qualitative analysis of trends in the literature.

\subsection{Document selection and review}

To assess if and how adaptation is occurring we identify and review peer reviewed publications documenting adaptation actions to climate change in developed nations, drawing upon recent methodological developments (Arnell 2010; Tompkins et al. 2010; Berrang-Ford et al. 2011). We make a distinction between the articulation of intentions or proposals to act, and adaptation actions themselves; we focus on the latter, with interventions requiring explicit recognition of climate change as a contributing motivator and involving the implementation of an intervention. Description of inclusion and exclusion criteria is provided in Supplementary Data (S2, S3). 
Relevant publications were identified from the global adaptation dataset created by Berrang-Ford et al. (2011), where a keyword search was performed in ISI Web of Knowledge (WOK) using the key topic terms "climat* chang*" AND "adapt*", focusing on literature published between 2006 and 2009. The initial search retrieved 1,741 documents (Supplementary Data S4), with 39 articles retained here for full review as profiling adaptation actions in developed nations. The inventory of publications included and excluded is provided in Supplementary Data (S5, S6), and is in searchable database form at: http://www.jamesford.ca/database. Included publications were assigned an identifier number (\#1-39), and we make reference to specific publications using this number.

To characterize adaptation actions a typology was utilized-building upon Smit et al. (1999, 2000)_-guided by the following key questions: (1) What is the stimulus motivating the adaptive response? (2) Who or what is adapting? and (3) What are the adaptation activities and outcomes? An extensive full-text review of all (39) articles was conducted using a typology questionnaire, which along with typology definitions is provided in Supplementary Data S7. The use of a questionnaire enabled quantitative analysis of key trends in adaptation reporting, allowing us to ask questions including: At what level are adaptations occurring? What are the primary adaptation responses? In which sectors is adaption most common? Is adaptation more likely to be proactive or reactive? In addition, an in-depth review of each article was conducted to provide contextual information on adaptation. This enabled us to ask questions including: are there concrete examples of adaptations that have been implemented and evaluated? How detailed is the information on interventions? Have stakeholders been involved in adaptation development? Is progress being made on mainstreaming? What constraining and enabling factors affect adaptation?

\subsection{Analysis}

Descriptive statistics (Stata) were used to summarize trends in the publications with categories guided by the typology questionnaire; inferential statistics were not feasible due to cell counts $(n=39)$. Summary data is provided in Supplementary Data S8. Qualitative analysis was performed using latent content analysis to identify key themes based upon description of adaptation actions within the selected publications. This involved firstly reading all the publications to discover patterns, themes, and categories. On this basis, coding categories were created organized around the themes established by the typology. Each article was read in-depth again and coded, whereby sections of text were manually assigned the appropriate code. Coded text was then retrieved, evaluated, and compared with the quantitative analysis to identify key characteristics of adaptation actions.

\section{Results}

3.1 Reporting on adaptation actions from developed nations is limited

Thirty nine publications (2\%) fit our inclusion criteria for intentional adaptation actions in developed nations. Excluded articles are largely associated with natural systems, vulnerability assessments that have not been translated to adaptation 
actions, or actions in developing nations (Supplementary Data S4). Over the observation period the number of publications reporting on adaptation actions has more than doubled (Fig. 1a). The depth of reporting, however, is limited. Only two publications in the dataset report on the development of adaptation plans $(\# 22,35)$ while many discuss adaptation actions briefly within the context of vulnerability studies.

\subsection{Higher-levels of government are playing a leading role in adaptations that are reported}

Adaptations are most frequently implemented at the municipal level, with $67 \%$ of articles reporting adaptations at this level, but also include regional (i.e. state or province) and national adaptations (Fig. 1b). Few articles document adaptations at the household level or report that non-governmental organizations or civil society are participating in adaptation activities. While adaptations were most frequently implemented municipally, higher-levels of government were the most likely to participate in adaptive governance (e.g. via funding, motivating adaptations; Fig. 1c). Correspondingly, institutional guidelines and governmental mechanisms were the dominant form of adaptation (Fig. 1d).

\subsection{Adaptations are most commonly documented in the transportation, infrastructure, and utilities sectors}

Reporting on adaptation interventions in the dataset is dominated by infrastructure, transportation, and utilities (Fig. 1e). Interventions in these sectors are typically motivated by their sensitivity to climate change impacts, particularly where decisions involve investments that have a long lifespan and will therefore be exposed to significantly different future conditions (e.g. engineering structures for coastal flood protection $(\# 21,25))$. The impacts of recent and historic extreme climate-related events have also served to create awareness in these sectors regarding their climate vulnerability stimulating the consideration of adaptation (e.g. \# 3, 6, 21, 22, 25). A small sub-set of publications $(16 \%)$ describe how adaptation forms part of larger mitigation activities (e.g. \#34, 35). Adaptations in the health sector were noted in only $21 \%$ of articles, while there is limited penetration of interventions into sectors not directly or traditionally associated with climate, including culture and education.

\subsection{Non-structural interventions are key components of adaptive response}

Non-structural interventions are noted in $90 \%$ of publications reviewed here, and involve developing management strategies, plans, policies, regulations, guidelines, or operating frameworks to guide current and/or future planning. Thirty eight percent of publications report adaptations involving structural interventions, and are typically preferred in cases where risks of climate change are acute, including coastal protection $(\# 13,21)$, transportation (\#3), municipal water supply (\#22), and permafrost thaw (\#1). Even here, however, non-structural interventions are increasing in importance. Penning-Roswell et al. (\#2), Carter et al. (\#36), and Urwin and Jordan (\#24) describe declining support for flood protection measures to reduce vulnerability in the UK in favour of land use planning and preparation. Adaptive 

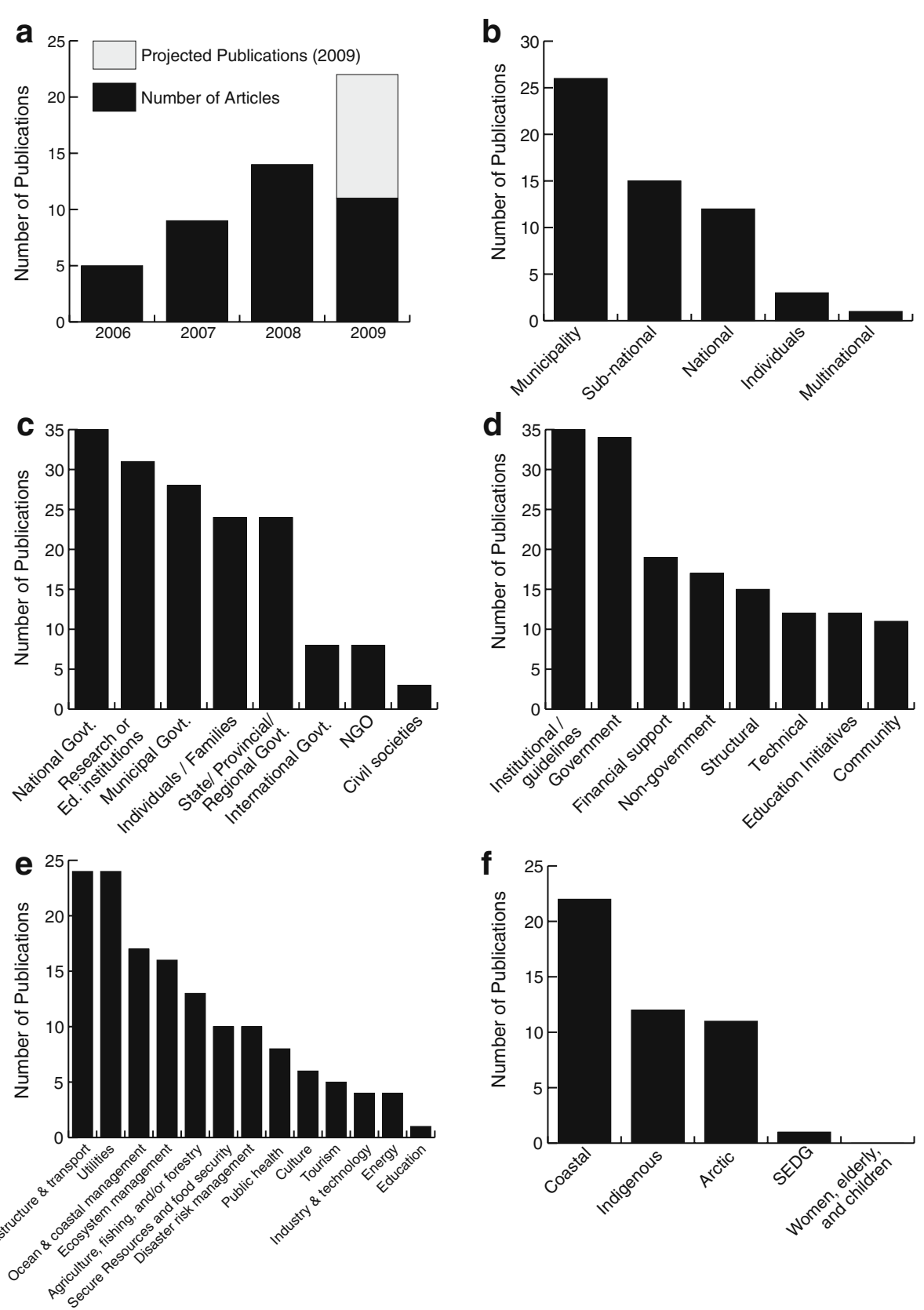

Fig. 1 a Number of publications reviewed, by year. The review included publication up to 1 July 2009 only, and therefore reflect only half of that year. A projection of the 2009 total publications (assuming homogeneous publication intensity throughout the year) has thus been added. b Spatial scale of adaptation. c Stakeholders involved in adaptation. d Adaptation mechanisms. e Sectors involved in adaptation. f Focus on vulnerable groups/regions. SEDG Socio-economically disadvantaged group. $W E C$ women, the elderly, and children 
management is emerging as a key component of non-structural adaptation, involving interventions to improve how human systems experience and deal with stress and change in general, bringing together multiple stakeholders (e.g. \#6). Financial support and education initiatives were mentioned by $31 \%$ and $28 \%$ of publications respectively.

\subsection{There is negligible consideration of vulnerable populations outside of the Arctic} and coastal areas

Adaptations in coastal systems and low-lying areas were the foci of half the publications, particularly studies from the UK and US (Fig. 1f). Articles profiling Indigenous adaptation were related to Arctic populations in Northern Canada and Alaska. There was only one report, from Australia, of adaptation involving nonArctic Indigenous populations. There were no reports on adaptation from nonArctic Indigenous populations in Canada, US, New Zealand, or Scandinavia. There is minimal reporting of socio-economically disadvantaged groups, and no articles focusing on adaptation action with regards to women, the elderly or children (Fig. 1f).

\subsection{Adaptation reporting differs by region}

Publications reporting adaptation actions are equally distributed between Europe and North America, with limited reporting from Australia and New Zealand. European adaptation publications are dominated by activities in the United Kingdom (8) and Holland (4). Close to one third of publications were associated with Arctic regions, all of which occurred in North America and all but one of which were associated with northern Indigenous populations.

\subsection{Adaptations reported in the dataset explicitly recognize future impacts}

Over $90 \%$ of publications report adaptations involving purposeful action where future climate change impacts are explicitly recognized, including efforts to enhance adaptive capacity and/or reduce sensitivity. This is consistent with the reported goals of adaptations, which are dominated by activities such as reducing risk, building partnerships, improving monitoring, strengthening institutions, enhancing research and promoting awareness. Adaptations of this nature are favoured in cases where there is a long lead in time to adaptation development necessitating complex negotiations, consultation, and planning; in cases where decisions made today have long term implications for climate-exposure (e.g. infrastructure, urban development; e.g. \#3, 6, 12, 21); and where vulnerability to climatic risks is high and/or historic events highlight vulnerability (e.g. \# 3, 6, 21, 22, 25). Despite this, there was a notable lack of evidence in the dataset of actions to capitalize on the potential benefits of climate change. This is reflective of reluctance among the research community in general to consider benefits of climate change, and might stem from the politicized nature of the climate debate where emphasis has focused on the need to push the issue of climate change onto the public and political agenda (Pielke 2010). 
3.8 Stakeholders are actively involved in adaptation actions reported here

The majority of publications report adaptation intervention to have involved stakeholders in developing and/or implementing adaptation actions. Seven articles (18\%) focus primarily on knowledge transfer and designing effective networks for engaging, working with and disseminating to stakeholders (\#5, 3, 7, 20, 26, 37, 39). Four of these publications are from a North American context, 3 from Arctic regions. A key aspect of Arctic adaptations involves the establishment of monitoring programmes integrating both science and traditional knowledge to facilitate safe use of sea ice environments (\#20,39). Reporting on UKCIP dominates the European publications on stakeholder involvement. Other articles explore approaches to knowledge translation and community involvement as secondary or tertiary objectives (e.g. \#6, $13,9,32)$.

\subsection{Multiple factors are motivating adaptation}

Climate change was reported as the sole reason for adaptation intervention in $26 \%$ of publications. The majority $(41 \%)$ involve climate change in combination with secondary factors as motivation, and in $33 \%$ of cases climate change has an equal or secondary role. Human systems adapting to climate change are undergoing multiple stresses, with limited resources constraining climate change specific interventions. Context is important, with adaptations typically motivated and/or enabled by broader developments. Hurricane Katrina, for instance, provided impetus for integrating climate change concerns into the management of water levels on Lake Okeechobee in Florida, with increasing emphasis on adaptive management enabling this development (\#6). Leadership is important. Norman (\#27) describes how the election of Prime Minister Rudd in Australia increased emphasis on all aspects of climate change, creating an enabling environment for the consideration of adaptation at national and regional levels. Saaverda and Budd (\#\#35) describe how links with the local university and forward thinking local leaders have made King Country an adaptation leader in the US, and a number of articles describe how UKCIP has been influential in promoting adaptation across the UK $(\# 5,26)$.

\subsection{Barriers to adaptation are widely noted}

Barriers to adaptation development and implementation are described in the majority of publications reviewed here. Institutional challenges are widely noted, often involving significant lead time to negotiate and consult with various interested parties. Vedwan et al. (\#6) offer insights from lake management in Florida where the development of more inclusive and responsive institutions capable of effectively integrating climate change has taken decades. Compounding the challenges of changing and creating institutions, political short-termism often limits the ability to plan for long term risks where benefits will be accrued in the future but political implications today. Few et al. (\#12) note this to be a major limit to coastal planning in the UK where local political necessity dictates that long term planning horizons promoted nationally are sacrificed where these goals conflict with other priorities. 
O'Brien et al. (\#4) caution that complacency towards climate change poses significant barriers in Europe, with a lack of awareness of potential dangers constraining action. Financial barriers, lack of information on the necessity to adapt, knowledge about available options, and the ability to implement most suitable ones, were not widely noted.

\section{Discussion}

Traditional literature reviews are useful for characterizing the broad contours of knowledge on a given area, but are less suited to answering specific questions such as posed here: are developed nations adapting to climate change? Questions of this nature require systematic review methodologies where explicit inclusion and exclusion criteria are developed to identify and analyze relevant literature. Herein, we develop and apply a mixed-methods review methodology, creating a database of publications reporting on adaptation actions in developed nations. We do not argue that the database captures all adaptation actions being implemented, with interventions likely reported in the grey literature and in articles published outside of the 2006-2009 review period. What the work does offer, however, is a proxy or indicator of adaptation action at national/regional levels - a snapshot of what is going on-that can be used to monitor adaptation over time, and is consistent with calls for more timely analyses to compliment IPCC (Oppenheimer et al. 2007). Importantly, examining how adaptation is and is not taking place enables us to offer a number of insights of relevance to research and policy.

Firstly, while adaptation is being thought about widely, examples of actual intervention reported in the literature are limited, raising concerns about the likelihood of effective adaptation given the speed of climate change and limited window of opportunity for action (Adger and Barnett 2009). This is compounded by the often significant time requirements for adaptation development to progress from problem identification to actual implementation, as identified in articles reviewed here. Where interventions are occurring we hypothesize that they represent action by early adopters, with adaptations typically at early stages of implementation. Thus no studies examined here formally assess success of interventions or consider how adaptations will perform under different climate scenarios. Adaptation reporting in unequal by region with actions only described in 14 annex- 1 countries. There is also no evidence in the dataset of adaptation actions targeting vulnerable populations including non-Arctic Indigenous populations, along with women, elderly, and children. Furthermore, while mainstreaming is evident, few studies describe the development of specific adaptation plans, which will be necessary in regions and sectors where future climatic conditions are expected to pose significant risks outside of those currently experienced (Füssel 2007).

Secondly, as adaptations reviewed here illustrate, high adaptive capacity typically assumed for developed nations will not necessarily translate into effective adaptation action. Thus - in addition to the concerns illustrated in the previous paragraph-a major challenge highlighted in a number of articles concerns the lack of political will to meaningfully address climate change impacts, particularly at local levels where a mismatch between national statements on adaptation and local action has been noted. In such cases adaptation interventions have preferred short term risk reduction over long term strategic planning, potentially increasing vulnerability and 
making future adaptation more difficult. Institutional barriers along with limited consideration of future climate scenarios in adaptation intervention, create potential for maladaptation.

The experience of adaptation reported here challenges complacency common among developed nations regarding their ability to adapt to climate change (Ford and Berrang-Ford 2011) and raises similar 'concerns' to those identified by Adger and Barnett (2009). Herein, continued monitoring of adaptation action is important and the approach developed here offers one way of achieving this. Additionally, the systematic review methodology holds insights for future climate change metaanalyses more broadly (e.g. IPCC AR5), specifically highlighting the importance of systematic, rigorous and transparent criteria for literature identification and selection. Otherwise, as recent debates over IPCC and 'climategate' highlight, it is easy for debates over science to be captured by the politicized nature of climate change (Pielke 2010). Systematic review methodologies-pioneered in the health sciences and developed here in a climate context - offer one such approach towards greater openness and rigor. It is also clear that further methodological developments are needed, specifically finding ways to systematically include grey literature. The challenges here are legion however: a search for "climate change and adapt" in Google documents over 2 million hits, the quality of grey literature is highly variable, and targeted inclusion and exclusion criteria and search terms are required to make reviews manageable. Notwithstanding, research in the health sciences offers guidelines on how to include grey literature and techniques to manage the search process (e.g. Cochrane Collaboration) and needs to be examined further for application in a climate change context.

With the climate change literature expanding rapidly and debates over the science and policy intervention becoming ever more polarized and politicized, meta-analyses can help us grasp what we know, don't know, and need to know. Continued methodological development and refinement herein needs to be a priority for future research.

Open Access This article is distributed under the terms of the Creative Commons Attribution Noncommercial License which permits any noncommercial use, distribution, and reproduction in any medium, provided the original author(s) and source are credited.

\section{References}

Adger WN, Barnett J (2009) Four reasons for concern about adaptation to climate change. Environ Plan A 41:2800-2805

Arnell NW (2010) Adapting to climate change: an evolving research programme. Clim Change 100:107-111

Berrang-Ford L et al (2011) Are we adapting to climate change? Glob Environ Change 21(1):25-33

Ford JD, Pearce T (2010) What we know, do not know, and need to know about climate change vulnerability in the western Canadian Arctic. Environ Res Lett 5. doi:10.1088/1748-9326/5/1/014008

Ford JD, Berrang-Ford L (2011) (eds) Climate change adaptation in developed nations: from theory to practice. Springer: Dordrecht, The Netherlands (in press)

Füssel HM (2007) Adaptation planning for climate change: concepts, assessment approaches, and key lessons. Sustain Sci 2(2):265-275

Hulme M (2009) Mapping climate change knowledge: an editorial essay. Wiley Interdisciplinary Reviews: Climate Change 1

Hulme M et al (2010) IPCC: cherish it, tweak it or scrap it? Nature 463:730-732 
New M et al (2011) Four degrees and beyond: the potential for a global temperature increase of four degrees and its implications. Philos Trans R Soc A 369(1934):6-19

Oppenheimer M et al (2007) Climate change-the limits of consensus. Science 317:1505-1506

Pielke R (2010) The climate fix: what scientists and politicians won't tell you about global warming. Basic, New York, p 276

Smit B et al (1999) The science of adaptation: a framework for assessment. Mitig Adapt Strategies Glob Chang 4:199-213

Smit B et al (2000) An anatomy of adaptation to climate change and variability. Clim Change 45:223251

Smith MS et al (2011) Rethinking adaptation for a 4 degrees C world. Philos Trans R Soc A 369(1934):196-216

Tompkins E et al (2010) Observed adaptation to climate change: UK evidence of transition to a well-adapting society. Glob Environ Change 20(4):627-635 